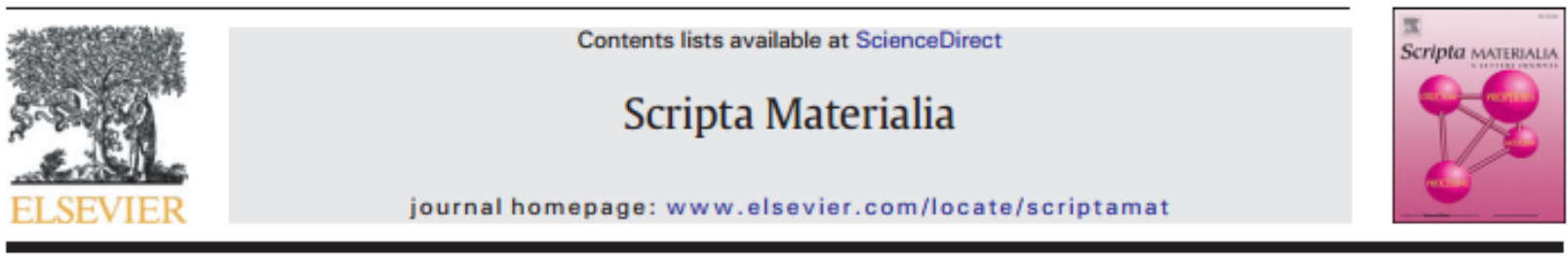

Regular Article

Determination of the critical stress for the initiation of dynamic transformation in commercially pure titanium

Clodualdo Aranas Jr. ${ }^{\mathrm{a}, *}$, Anes Foul ${ }^{\mathrm{a}}$, Baoqi Guo ${ }^{\mathrm{a}}$, Ameth Fall ${ }^{\mathrm{b}}$, Mohammad Jahazi ${ }^{\mathrm{b}}$, John J. Jonas ${ }^{\mathrm{a}}$

4 Materials Engineering, MoGill University, 3610 University St, Montreal H3A OCS, Canoda

b Département de Génie Mécanique, École de Technologie Supérieure, 1100 nue Notre-Dame Ouest, Montreal H3C 1K3, Canada

Authors' accepted manuscript

Article published in Scripta Materialia, vol. 133 (2017)

https://doi.org/10.1016/j.scriptamat.2017.02.022

(c) 2017. Made available under the CC-BY-NC-ND 4.0 license

http://creativecommons.org/licenses/by-nc-nd/4.0/ 


\title{
Determination of the critical stress for the initiation of dynamic transformation in commercially pure titanium
}

Clodualdo Aranas Jr. ${ }^{1}$, Anes Foul ${ }^{1}$, Baoqi Guo ${ }^{1}$, Ameth Fall ${ }^{2}$, Mohammad Jahazi ${ }^{2}$ and John J. Jonas ${ }^{1}$

${ }^{1}$ Materials Engineering, McGill University 3610 University St., Montreal, Canada H3A 0C5

${ }^{2}$ Département de Génie Mécanique, École de Technologie Supérieure 1100 rue Notre-Dame Ouest, Montreal, Canada H3C 1K3

\begin{abstract}
The dynamic transformation of alpha to beta is shown to take place in titanium at temperatures below the beta transus. The driving force for this transformation is the net softening associated with the formation of the lower flow stress beta phase. The obstacle to the transformation consists of the free energy difference between the phases as well as the work of dilatation during the phase change. Here the critical condition for transformation is defined as the moment when the driving force becomes equal to the obstacle energy. This approach is supported by data obtained from compression tests.
\end{abstract}

Keywords: dynamic transformation, transformation softening, beta $\mathrm{Ti}$

In the early 2000s, Koike and co-workers showed that some of the alpha phase of a titanium alloy transforms into beta when it is deformed below the beta transus temperature [1]. In their 
work, they observed significant increases in the beta volume fraction when samples of Ti$5.5 \mathrm{wt} \% \mathrm{Al}-1 \mathrm{wt} \% \mathrm{Fe}$ were strained in tension at temperatures in the range $777^{\circ} \mathrm{C}$ to $927^{\circ} \mathrm{C}$. For reference, the beta transus temperature of their alloy was $997^{\circ} \mathrm{C}$. They accounted for their observations by proposing that the free energy of the alpha increased by about $500 \mathrm{~J} / \mathrm{mol}$ as a result of straining, although they did not suggest the source of this increase. Since this first report, other researchers have now provided evidence of such dynamic transformation in other titanium alloys [2-4].

More recently, the present authors have proposed that these phase changes are propelled by the transformation softening that accompanies the changes [5]. This type of analysis was initially derived to explain the dynamic transformation (DT) that takes place when steels are deformed above the $\mathrm{Ae}_{3}$ temperature [6]. Here the relatively hard high temperature austenite is replaced by the much softer low temperature ferrite phase. This method of analysis has now been extended to the case of titanium and its alloys, where the deformation is applied below the beta transus. This difference arises because the high temperature beta is softer than the low temperature alpha phase (whereas in steels the high temperature phase is harder than the low temperature phase).

According to the transformation softening model $[6,7]$, the net softening during transformation is the driving force for the phase change. This can be evaluated by taking the difference between the critical stress for transformation in the harder phase and the yield stress of the softer phase that takes its place. In steels, the critical stresses are usually determined by double differentiation of the flow curves [8]. Unfortunately, this type of approach is not well suited to 
titanium alloy flow curves (e.g. Ti-64 and IMI-834) because of their high work hardening rates (high slopes). An alternative method has therefore been developed for such cases, which is described below. It is based on the concept that the transformation is initiated when the driving force becomes equal to the obstacle energy, as given by:

$$
E_{D F}=E_{B}
$$

where $E_{D F}$ is the driving force and $E_{B}$ is the energy of the barrier to the transformation. $B y$ substituting equations for the driving force and the obstacle [6], the above equation can be expressed as:

$$
\sigma_{C}-\sigma_{\beta-Y S}=\Delta G_{\alpha-\beta}+(W / V)_{D}+(W / V)_{S A}
$$

Here $\sigma_{C}$ is the critical stress for transformation, $\sigma_{\beta-\gamma s}$ is the yield stress of the beta as evaluated by extrapolation to below the beta transus temperature, $\Delta G_{\alpha-\beta}$ is the free energy difference between the alpha and beta, $(\mathrm{W} / \mathrm{V})_{\mathrm{D}}$ is the work per unit volume associated with dilatation and $(\mathrm{W} / \mathrm{V})_{S A}$ is the work per unit volume due to shear accommodation. $\Delta \mathrm{G}_{\alpha-\beta}$ can be readily determined by using the FactSage thermodynamic software and its dependence on temperature calculated in this way is displayed in Fig. $\mathbf{1 .}$

In the next section, the method is demonstrated for two temperatures, namely, $850^{\circ} \mathrm{C}$ and 875 ${ }^{\circ} \mathrm{C}$, which are $43^{\circ} \mathrm{C}$ and $18{ }^{\circ} \mathrm{C}$ below the transus temperature, respectively. Here transformation at the lower temperature requires the provision of $159 \mathrm{~J} / \mathrm{mol}$ of energy, while the higher temperature only needs about $65 \mathrm{~J} / \mathrm{mol}$ of additional free energy. It is also important to note that continued decreases in temperature continue to increase the height of the energy barrier 
until finally transformation becomes too difficult to initiate. This is because the driving force does not increase rapidly enough with decreasing temperature. Such behavior is in sharp contrast to that of steels, where the Gibbs energy difference displays a peak at intermediate temperatures because ferrite is stable both at low (alpha) and high (delta) temperatures [9].

The transformation of alpha to beta is accompanied by a dilatation strain of $1.7 \%$ [10]. The work of dilatation can therefore be expressed by the equation:

$$
(W / V)_{D}=\sigma_{C} \times 0.707 \times 0.017
$$

where 0.707 is the orientation factor $\left(\sin \theta, \theta=45^{\circ}\right)$ associated with the maximum resolved shear stress, i.e. with the habit plane of the transformation. This value is based on the assumption that the alpha grains with the highest Schmid factors (most suitably oriented grains with respect to the applied load) will be the first grains to transform during straining. Although the transformation also requires shear accommodation, this has been shown to be negligible and so is not included in the present calculations [11].

The yield stress of the single phase beta ( $\left.\sigma_{\beta-\gamma s}\right)$ in eq. $\mathbf{2}$ cannot be measured experimentally below the transus, of course. Nevertheless, estimates of this quantity were made by carrying out compression tests above the beta transus (not shown here) and extrapolating the values to temperatures below the transus. In terms of these values, the critical stress for initiating the transformation is calculated by substituting eq. $\mathbf{3}$ into eq. $\mathbf{2}$, as given by:

$$
\sigma_{C}-\sigma_{\beta-Y S}=\Delta G_{\alpha-\beta}+\sigma_{C} \times 0.707 \times 0.017
$$

and 


$$
\sigma_{C}=\frac{\Delta G_{\alpha-\beta}+\sigma_{\beta-Y S}}{[1-(0.707)(0.017)]}
$$

The dependence of the critical stress on temperature defined in this way is shown in Fig. 2 a. Here the critical stresses at $850^{\circ} \mathrm{C}$ and $875^{\circ} \mathrm{C}$ are about $22 \mathrm{MPa}$ and $14 \mathrm{MPa}$, respectively. Note that once the critical stress is attained, the amount of beta transformed increases progressively with increasing stress and strain. This type of diagram can also be used to predict the lowest possible temperature at which the driving force is large enough to transform alpha into beta. Note that the critical stresses obtained from the conventional double differentiation method are associated with softer phase volume fractions of $\sim 5 \%$, as this amount must be formed in order for its effect on the work hardening to be detectable [6]. As a result, the critical stresses predicted in this way are expected to be somewhat higher than the ones obtained from the present method.

Once the critical stress has been identified, the work of dilatation can be deduced. This is illustrated in Fig. 2b. Here the required amount of work increases with decreasing temperature and is in the range $1-8 \mathrm{~J} / \mathrm{mol}$ at temperatures close to the beta transus.

As discussed above, the driving force for transformation is the net softening defined as the difference between the critical stress and the yield stress of the fresh beta phase. The dependences of the critical and yield stresses on inverse absolute temperature are displayed in Fig. 3. Here the driving force to initiate transformation is shown to be about $573 \mathrm{~J} / \mathrm{mol}$ when the temperature has decreased to $143{ }^{\circ} \mathrm{C}$ below the transus. Conversely, this is reduced to about $162 \mathrm{~J} / \mathrm{mol}$ when the temperature is only $43^{\circ} \mathrm{C}$ below the transus. 
The method described above is illustrated using the results of compression tests carried out on C.P. Ti grade 2 at $850^{\circ} \mathrm{C}$ and $875{ }^{\circ} \mathrm{C}$ displayed in Fig. 4. Here a strain of 0.75 was applied at a strain rate of $0.01 \mathrm{~s}^{-1}$. The curves were first fitted with a $9^{\text {th }}$ order polynomial; this was followed by the application of the double differentiation method (not shown here), as described in Ref.

8. Two softening mechanisms were observed to be initiated, with critical stresses of 23 and 27 MPa in the first set and 24 and $29 \mathrm{MPa}$ in the second (marked with solid circles). The first set of critical stresses is considered here to apply to the initiation of DT while the second to DRX [6].

The critical stresses obtained from the total energy barrier and double differentiation methods are listed in Table 1. Generally, the former provides the exact moment that transformation begins while the latter overestimates the critical stress since it requires the formation of about $5 \%$ of the dynamic phase in order for the operation of the softening mechanism to be detectable.

The transformation softening model was employed here to predict the initiation of dynamic transformation in pure Ti alloy below the beta transus temperature. This involved defining the driving force as the difference between the critical stress for transformation in the harder phase (alpha) and the yield stress of the softer phase (beta) that takes its place. The present observations can be summarized as follows:

1. The critical stress for DT can be determined by equating the total energy obstacle to the driving force. The energy barrier consists of the free energy difference between beta and alpha and the work of dilatation associated with the transformation. Here the work of shear accommodation during transformation is considered to be negligible. 
2. The critical stresses obtained from the energy barrier method provide the exact moment of initiation of DT. The double differentiation method overestimates the critical stress since significant amounts of the dynamic phase must be present in order for its effect on the work hardening rate to be detectable.

The authors acknowledge with gratitude funding received from the McGill Engineering Doctoral Award (MEDA) program (CAJr), the Industrial Research Chair in Forming Technologies of High

Strength Materials of the École de Technologie Supérieure of Montréal (AF), and the Natural Sciences and Engineering Research Council of Canada.

\section{References}

[1] J. Koike, Y. Shimoyama, I. Ohnuma, T. Okamura, R. Kainuma, K. Ishida, K. Maruyama, Acta Mater. 48 (2000) 2059-2069.

[2] C. Xu, W. Zhu, Trans. Nonferrous Met. Soc. China 20 (2010) 2162-2167.

[3] H. Yang, G. Gurewitz, A. Mukherjee, Materials T. JIM 32 (1991) 465-472.

[4] Y. Zong, D. Shan, M. Xu, Y. Lv, J. Mater. Process. Technol. 209 (2009) 1988-1994.

[5] J.J. Jonas, C. Aranas Jr., A. Fall, M. Jahazi, Mater. Des. 113 (2017) 305-310.

[6] C. Ghosh, C. Aranas, J.J. Jonas, Prog. Mater. Sci. 82 (2016) 151-233.

[7] C. Aranas, J.J. Jonas, Acta Mater. 82 (2015) 1-10.

[8] E. Poliak, J. J. Jonas, Acta Mater. 44 (1996) 127-136.

[9] R. Grewal, C. Aranas Jr., K. Chadha, D. Shahriari, M. Jahazi, J.J. Jonas, Acta Mater. 109 (2016) 23-31. 
[10] G.A. Sargent, K.T. Kinsel, A.L. Pilchak, A.A. Salem, S.L. Semiatin, Metall. Mater. Trans. A 43 (2012) 3570-3585.

[11] A. Kelly, K.M. Knowles, Crystallography and Crystal Defects, $2^{\text {nd }}$ ed, John Wiley \& Sons, Ltd., United Kingdom, 2012.

\section{Figure Captions}

Figure 1. Free energy difference between the alpha and beta phases in pure titanium. Here the Gibbs energy differences are 159 and $65 \mathrm{~J} /$ mole at temperatures $43{ }^{\circ} \mathrm{C}$ and $18{ }^{\circ} \mathrm{C}$ below the transus temperature, respectively.

Figure 2. a) Dependence of the critical stress on temperature predicted by the energy barrier method. b) Dependence on temperature of the work of dilatation during transformation.

Figure 3. Dependences on inverse absolute temperature of the critical stress and the $\beta$-yield stress. The driving force for transformation is the difference between the critical stress and the $\beta$-yield stress.

Figure 4. Stress-strain curves determined on the C.P. Ti Grade 2 alloy using compression testing at a strain rate of $0.01 \mathrm{~s}^{-1}$. The solid circles are the critical stresses obtained from the double differentiation method. 


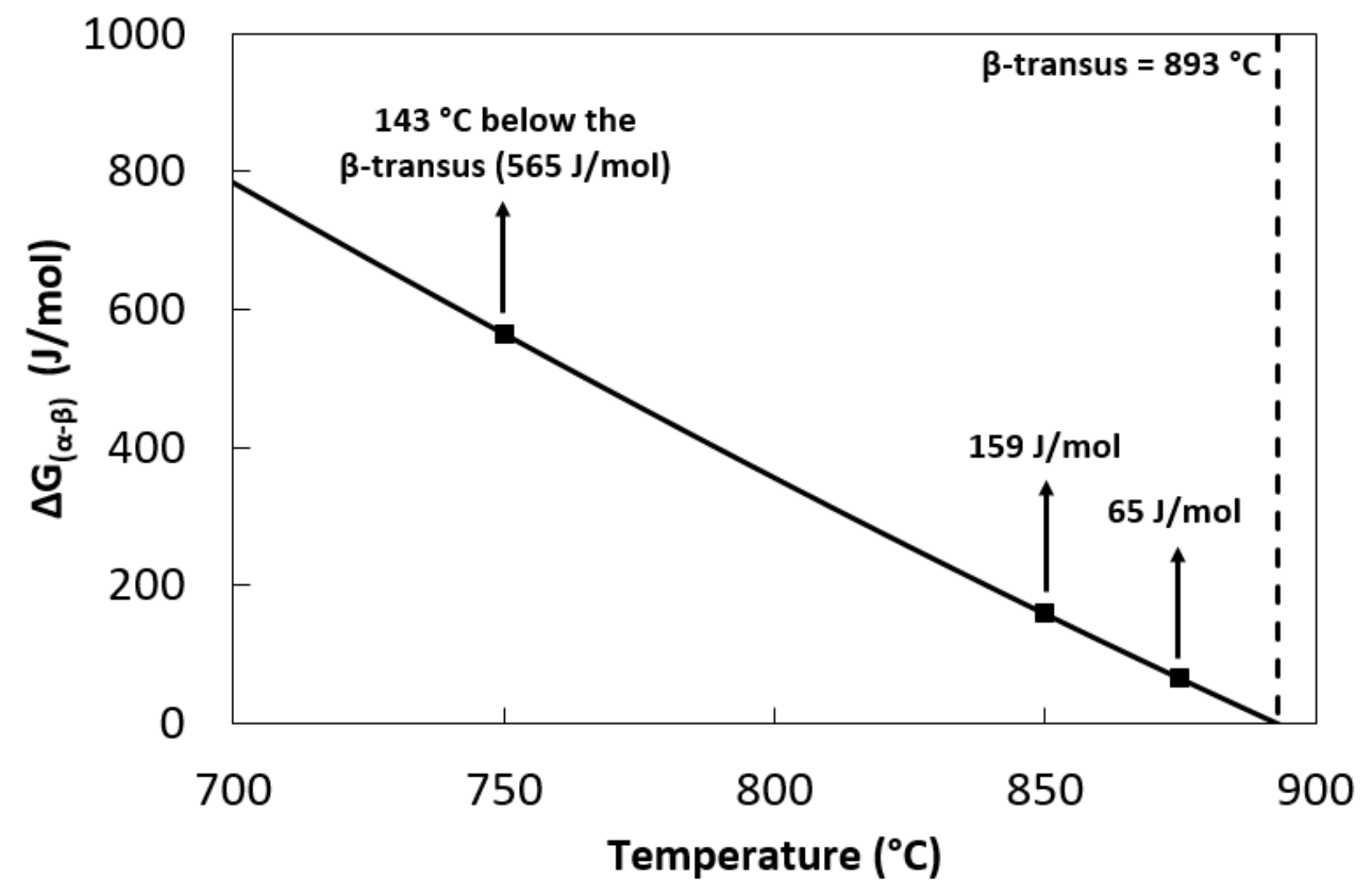

Figure 1. Free energy difference between the alpha and beta phases in pure titanium. Here the Gibbs energy differences are 159 and $65 \mathrm{~J} / \mathrm{mole}$ at temperatures $43{ }^{\circ} \mathrm{C}$ and $18{ }^{\circ} \mathrm{C}$ below the transus temperature, respectively. 

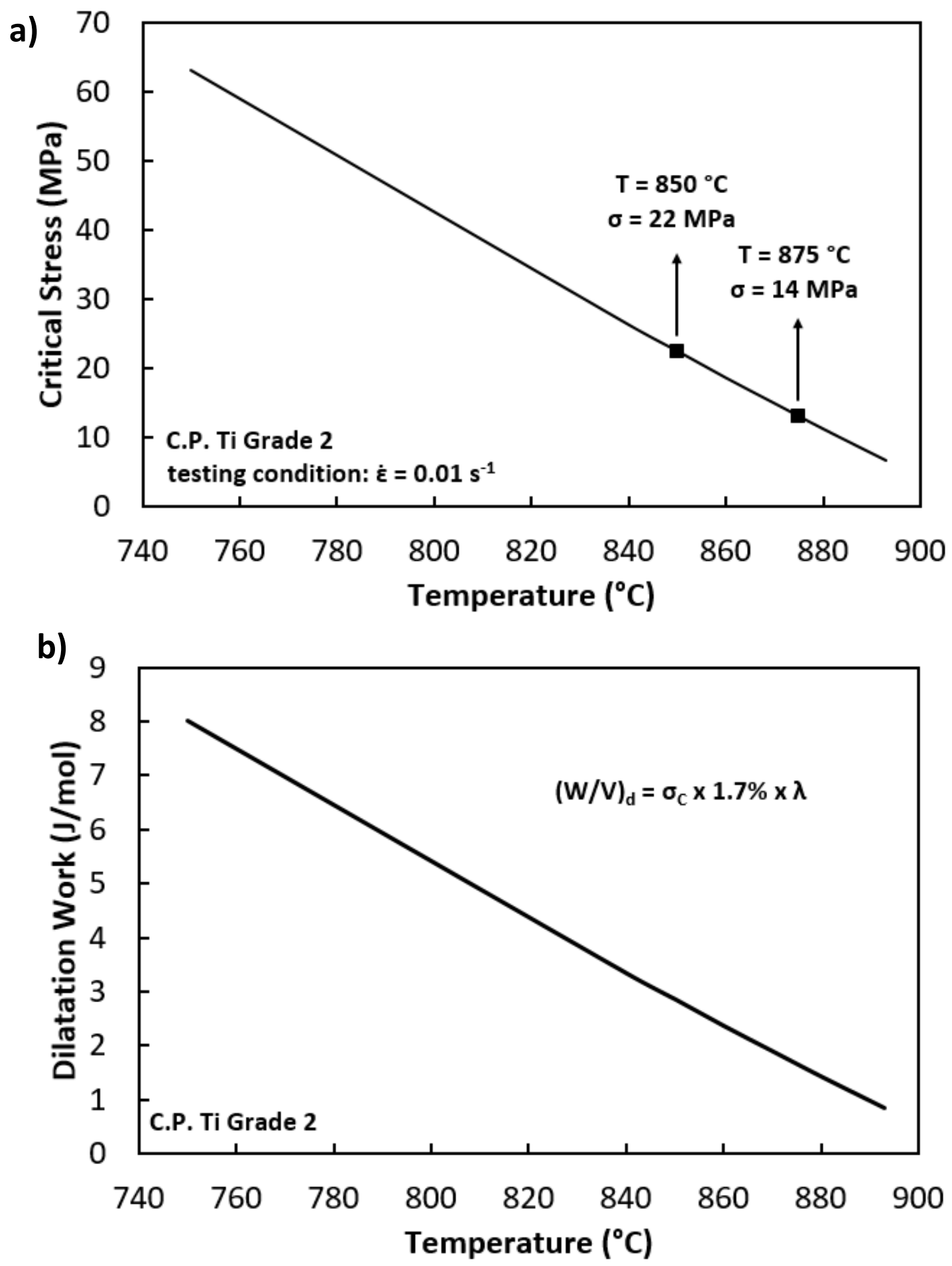

Figure 2. a) Dependence of the critical stress on temperature predicted by the energy barrier method. b) Dependence on temperature of the work of dilatation during transformation. 


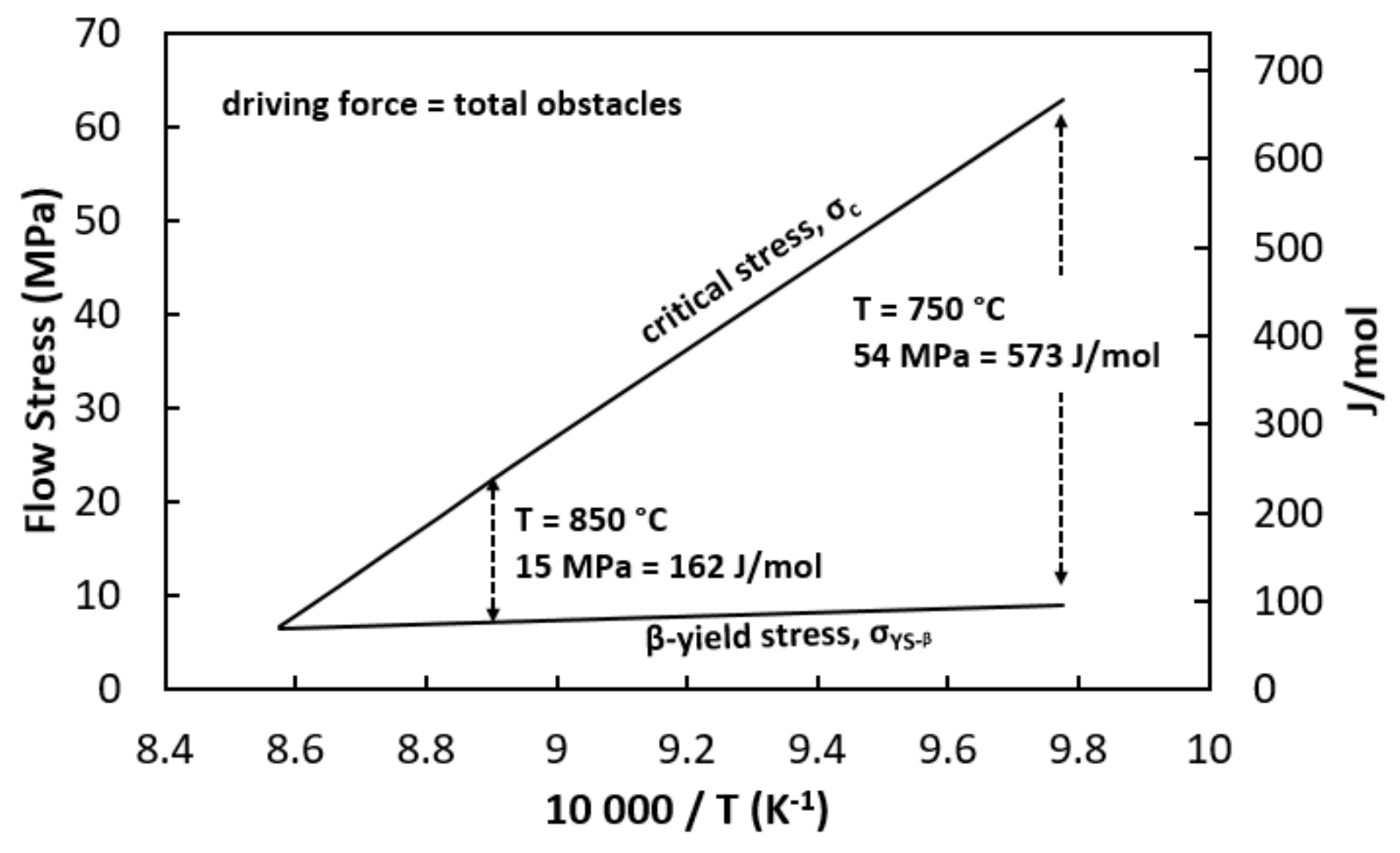

Figure 3. Dependences on inverse absolute temperature of the critical stress and the $\beta$-yield stress. The driving force for transformation is the difference between the critical stress and the $\beta$-yield stress. 


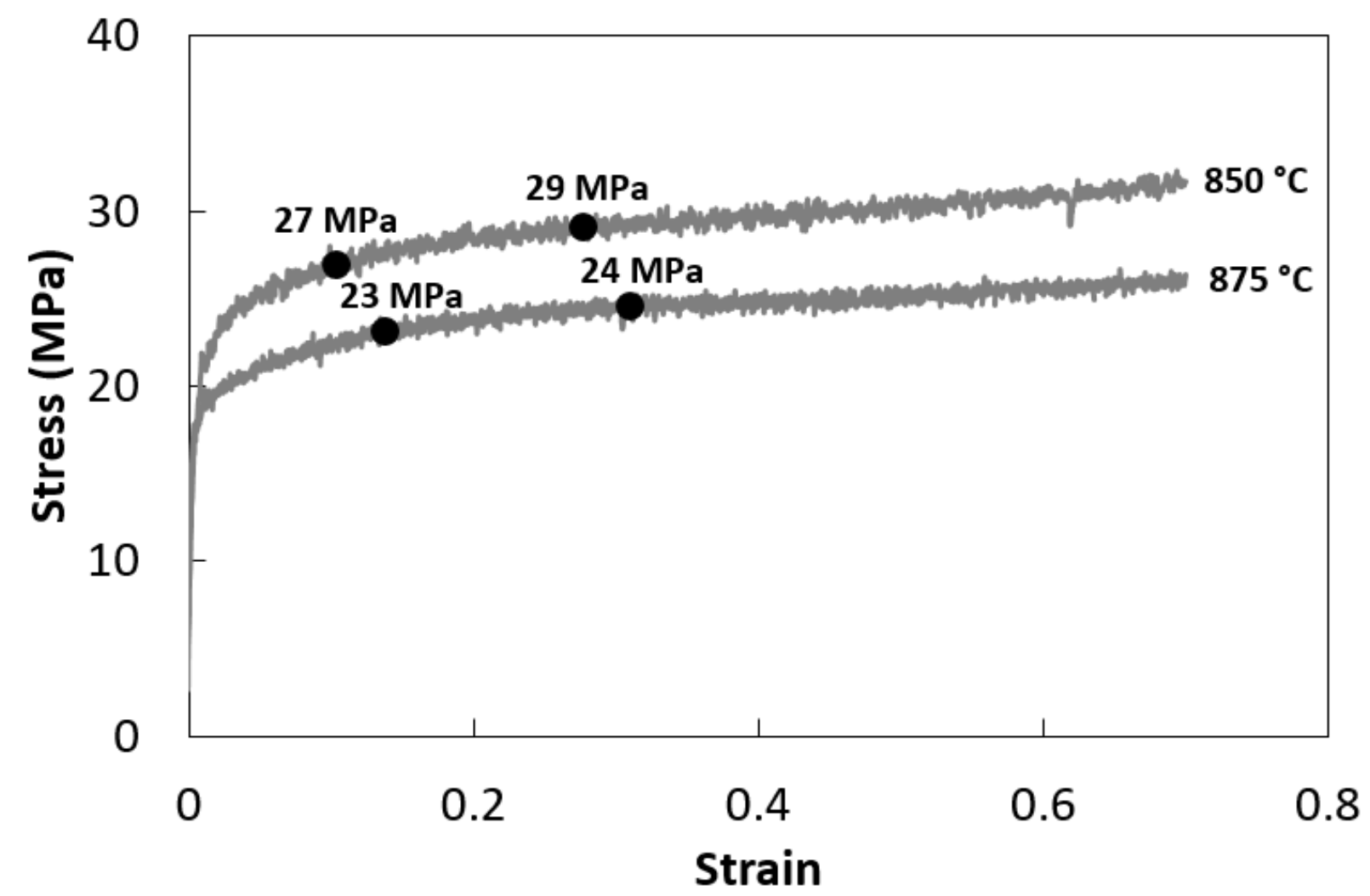

Figure 4. Stress-strain curves determined on the C.P. Ti Grade 2 alloy using compression testing at a strain rate of $0.01 \mathrm{~s}^{-1}$. The solid circles are the critical stresses obtained from the double differentiation method. 
Table 1. Comparison between the critical stresses obtained using double differentiation and the energy barrier method.

\begin{tabular}{ccc}
\hline \multirow{2}{*}{ Temperature } & \multicolumn{2}{c}{ Critical Stress } \\
\cline { 2 - 3 } & Double Differentiation & Energy Barrier \\
\hline $850^{\circ} \mathrm{C}$ & $27 \mathrm{MPa}$ & $22 \mathrm{MPa}$ \\
$875^{\circ} \mathrm{C}$ & $23 \mathrm{MPa}$ & $14 \mathrm{MPa}$ \\
\hline
\end{tabular}



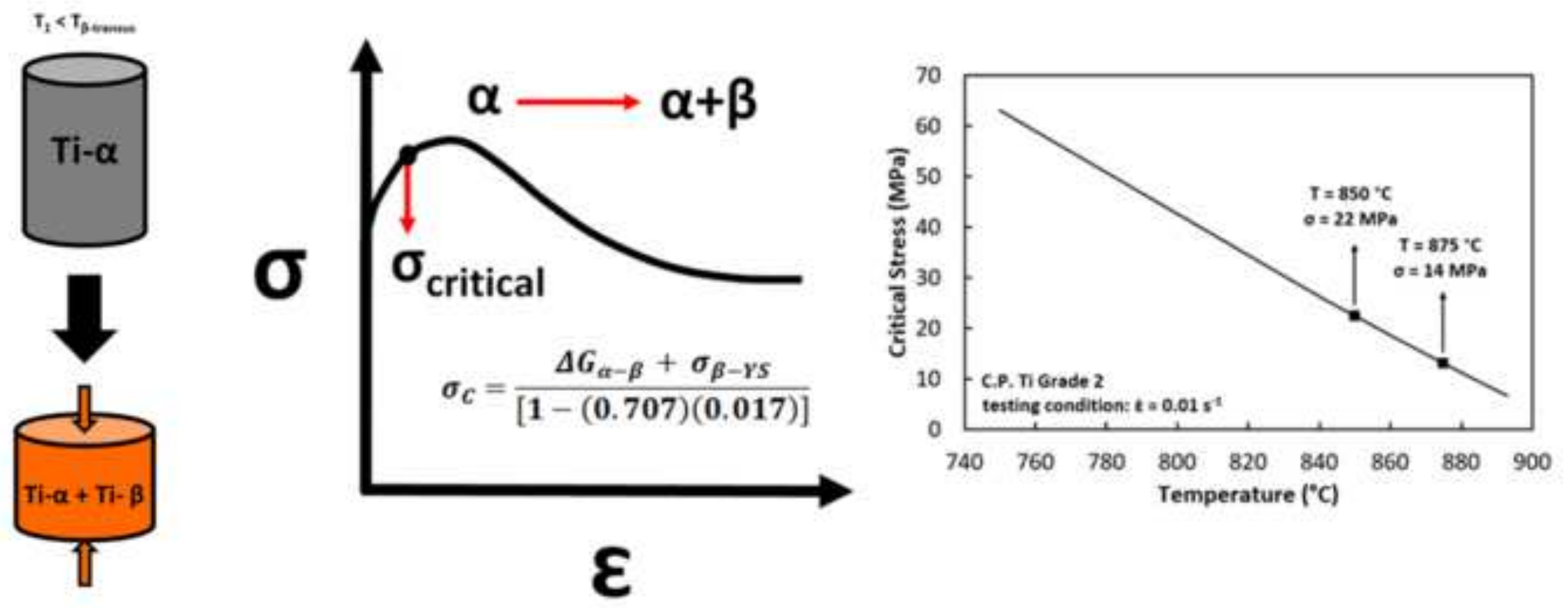